\title{
Weighted Estimates for the Maximal Operator of a Multilinear Singular Integral
}

by

\author{
Xi CHEN
}

\author{
Presented by Stanisław KWAPIEŃ
}

Summary. An improved multiple Cotlar inequality is obtained. From this result, weighted norm inequalities for the maximal operator of a multilinear singular integral including weak and strong estimates are deduced under the multiple weights constructed recently.

1. Introduction. Grafakos and Torres [4] systematically studied multilinear Calderón-Zygmund singular integral operators $T: \mathcal{S}\left(\mathbb{R}^{n}\right) \times \cdots \times$ $\mathcal{S}\left(\mathbb{R}^{n}\right) \rightarrow \mathcal{S}^{\prime}\left(\mathbb{R}^{n}\right)$ with some boundedness properties, defined by

$$
T\left(f_{1}, \ldots, f_{m}\right)(x)=\int_{\left(\mathbb{R}^{n}\right)^{m}} K\left(x, y_{1}, \ldots, y_{m}\right) f_{1}\left(y_{1}\right) \cdots f_{m}\left(y_{m}\right) d y_{1} \cdots d y_{m},
$$

where $K\left(x, y_{1}, \ldots, y_{m}\right)$ is a locally integrable function supported away from the diagonal $x=y_{1}=\cdots=y_{m}$ in $\left(\mathbb{R}^{n}\right)^{m+1}$ and satisfies

(i) (Size estimate)

$$
\left|K\left(x, y_{1}, \ldots, y_{m}\right)\right| \leq \frac{A}{\left(\left|x-y_{1}\right|+\cdots+\left|x-y_{m}\right|\right)^{m n}}
$$

for some $A>0$ and all $\left(x, y_{1}, \ldots, y_{m}\right) \in\left(\mathbb{R}^{n}\right)^{m+1}$ with $x \neq y_{j}$ for some $j$;

2010 Mathematics Subject Classification: 42B20, 42B25.

Key words and phrases: multilinear operator, maximal operator of singular integral, multiple weights, weighted norm inequalities. 
(ii) (Smoothness estimates)

$$
\begin{aligned}
\left|K\left(x, y_{1}, \ldots, y_{m}\right)-K\left(x^{\prime}, y_{1}, \ldots, y_{m}\right)\right| & \\
& \leq \frac{A\left|x-x^{\prime}\right|^{\epsilon}}{\left(\left|x-y_{1}\right|+\cdots+\left|x-y_{m}\right|\right)^{m n+\epsilon}}
\end{aligned}
$$

for some $\epsilon>0$ whenever $\left|x-x^{\prime}\right| \leq \frac{1}{2} \max _{1 \leq j \leq n}\left|x-y_{j}\right|$, and also, for each $j$,

$$
\begin{aligned}
\mid K\left(x, y_{1}, \ldots, y_{j}, \ldots, y_{m}\right)-K( & \left.x, y_{1}, \ldots, y_{j}^{\prime}, \ldots, y_{m}\right) \mid \\
& \leq \frac{A\left|y_{j}-y_{j}^{\prime}\right|^{\epsilon}}{\left(\left|x-y_{1}\right|+\cdots+\left|x-y_{m}\right|\right)^{m n+\epsilon}}
\end{aligned}
$$

for some $\epsilon>0$ whenever $\left|y_{j}-y_{j}^{\prime}\right| \leq \frac{1}{2} \max _{1 \leq j \leq n}\left|x-y_{j}\right|$.

In [5], the authors considered the corresponding maximal operator defined as

$$
T^{*}\left(f_{1}, \ldots, f_{m}\right)(x)=\sup _{\delta>0}\left|T_{\delta}\left(f_{1}, \ldots, f_{m}\right)(x)\right|,
$$

where $T_{\delta}$, the truncated operator of $T$, is

$$
\begin{aligned}
T_{\delta}\left(f_{1}, \ldots, f_{m}\right)(x) & \\
& =\int_{\left|x-y_{1}\right|^{2}+\cdots+\left|x-y_{m}\right|^{2}>\delta^{2}} K\left(x, y_{1}, \ldots, y_{m}\right) f_{1}\left(y_{1}\right) \cdots f_{m}\left(y_{m}\right) d y_{1} \cdots d y_{m} .
\end{aligned}
$$

Similarly to the linear setting, Cotlar's inequality, for all $\eta>0$,

$$
T^{*}\left(f_{1}, \ldots, f_{m}\right)(x) \leq C\left(M_{\eta}\left(T\left(f_{1}, \ldots, f_{m}\right)\right)(x)+\prod_{i=1}^{m} M f_{i}(x)\right),
$$

where $\left.M_{\eta}(f)(x)=\sup _{Q \ni x}|Q|^{-1} \int_{Q}|f|^{\eta}\right)^{1 / \eta}$ was employed to show

TheOrEm 1.1 (Boundedness of $T^{*},[5]$ ). Assume that $1 / p=1 / p_{1}+\cdots+$ $1 / p_{m}$.

(i) If $1<p_{1}, \ldots, p_{m} \leq \infty$ and $p<\infty$, then

$$
T^{*}: L^{p_{1}}\left(\mathbb{R}^{n}\right) \times \cdots \times L^{p_{m}}\left(\mathbb{R}^{n}\right) \rightarrow L^{p}\left(\mathbb{R}^{n}\right) .
$$

(ii) If $1 \leq p_{1}, \ldots, p_{m} \leq \infty$ and $p<\infty$, then

$$
T^{*}: L^{p_{1}}\left(\mathbb{R}^{n}\right) \times \cdots \times L^{p_{m}}\left(\mathbb{R}^{n}\right) \rightarrow L^{p, \infty}\left(\mathbb{R}^{n}\right) .
$$

By generalizing Coifman and Fefferman's good- $\lambda$ inequality of [1], Grafakos and Torres proved a weighted norm inequality for $T^{*}$ :

THEOREM 1.2 (Weighted boundedness of $T^{*},[5]$ ). Assume that $1<$ $p_{1}, \ldots, p_{m}<\infty, p_{0}=\min \left(p_{1}, \ldots, p_{m}\right)$ and $1 / p=1 / p_{1}+\cdots+1 / p_{m}$. If $\omega \in A_{p_{0}}$, then

$$
T^{*}: L^{p_{1}}(\omega) \times \cdots \times L^{p_{m}}(\omega) \rightarrow L^{p}(\omega) .
$$


Recently, Lerner, Ombrosi, Pérez, Torres and Trujillo-González [6] constructed a new theory of multiple $A_{\vec{p}}$ weights.

Definition 1.1 (Multiple weights, [6]). Let $1 \leq p_{1}, \ldots, p_{m}<\infty$ and $1 / p=1 / p_{1}+\cdots+1 / p_{m}$. Given $\vec{\omega}=\left(\omega_{1}, \ldots, \omega_{m}\right)$, set $\nu_{\vec{\omega}}=\prod_{i=1}^{m} \omega_{i}^{p / p_{i}}$. By definition, $\vec{\omega} \in A_{\vec{p}}$ if and only if

$$
\sup _{Q}\left(\frac{1}{|Q|} \int_{Q} \nu_{\vec{\omega}}\right)^{1 / p} \prod_{i=1}^{m}\left(\frac{1}{|Q|} \int_{Q} \omega_{i}^{1-p_{i}^{\prime}}\right)^{1 / p_{i}^{\prime}}<\infty .
$$

When $p_{i}=1,\left(|Q|^{-1} \int_{Q} \omega_{i}^{1-p_{i}^{\prime}}\right)^{1 / p_{i}^{\prime}}$ is understood as $\left(\inf _{Q} \omega_{i}\right)^{-1}$.

A more subtle multilinear maximal operator $\mathcal{M}$ which is defined as

$$
\mathcal{M}\left(f_{1}, \ldots, f_{m}\right)(x)=\sup _{Q} \prod_{i=1}^{m} \frac{1}{|Q|} \int_{Q}\left|f_{i}\right|
$$

was investigated to characterize the multiple weights in [6]. The authors showed that $\vec{\omega} \in A_{\vec{p}}$ is equivalent to either of the two weighted estimates for $\mathcal{M}$ :

(i) If $1<p_{1}, \ldots, p_{m}<\infty$ and $1 / p=1 / p_{1}+\cdots+1 / p_{m}$, then

$$
L^{p_{1}}\left(\omega_{1}\right) \times \cdots \times L^{p_{m}}\left(\omega_{m}\right) \rightarrow L^{p}\left(\nu_{\vec{\omega}}\right) .
$$

(ii) If $1 \leq p_{1}, \ldots, p_{m}<\infty$ and $1 / p=1 / p_{1}+\cdots+1 / p_{m}$, then

$$
L^{p_{1}}\left(\omega_{1}\right) \times \cdots \times L^{p_{m}}\left(\omega_{m}\right) \rightarrow L^{p, \infty}\left(\nu_{\vec{\omega}}\right) .
$$

Further, it was proved in [6] that $T$ satisfies both $(1.5)$ and $(1.6)$ by using unweighted boundedness, Fefferman-Stein inequalities and a sharp estimate. For more details, the readers are referred to [6].

It is natural to ask whether (1.5) and (1.6) hold for $T^{*}$. We will give a positive answer in this note. Instead of the good- $\lambda$ inequality we will employ Cotlar's inequality as in [2, p. 147]. However, $\prod_{i=1}^{m} M f_{i}$ fails to satisfy either (1.5) or (1.6) (see [6]), which makes us improve (1.4) by replacing the $m$-fold product of $M$ with $\mathcal{M}$. After the modification, we shall obtain not only strong type bounds but also weak endpoint estimates.

Theorem 1.3 (Weighted estimates for $T^{*}$ ). Assume that $1 / p=1 / p_{1}+$ $\cdots+1 / p_{m}$ and $\vec{\omega} \in A_{\vec{p}}$. Then both (1.5) and (1.6) hold for $T^{*}$.

Throughout this article, we write $\vec{f}=\left(f_{1}, \ldots, f_{m}\right), \vec{y}=\left(y_{1}, \ldots, y_{m}\right)$ and $\int_{Q^{m}} \prod_{i=1}^{m} f_{i}\left(y_{i}\right) d y_{i}=\int_{Q} \cdots \int_{Q} f_{1}\left(y_{1}\right) \cdots f_{m}\left(y_{m}\right) d y_{1} \cdots d y_{m}$ for convenience.

2. Weighted norm inequalities. The multiple $A_{\vec{p}}$ weights are appropriate for the maximal function $\mathcal{M}(\vec{f})$ which is more refined than $\prod_{i=1}^{m} M f_{i}$. Hence, we improve Cotlar's inequality as follows. 
Lemma 2.1 (Improved Cotlar inequality). For any $\eta>0$, there is a $C>0$ depending on $\eta$ such that

$$
T^{*}(\vec{f})(x) \leq C\left(M_{\eta}(T(\vec{f}))(x)+\mathcal{M}(\vec{f})(x)\right),
$$

where $M_{\eta}(f)(x)=\sup _{Q \ni x}\left(|Q|^{-1} \int_{Q}|f|^{\eta}\right)^{1 / \eta}$.

Proof. The basic idea is due to [5] and [6].

Fix $x \in \mathbb{R}^{n}, 0<\eta<1 / m$ and $\delta>0$. Denote by $Q(x, \delta)$ the cube of center $x$ and edge length $2 \delta$ with sides parallel to the axes, and set $U_{\delta}(x)=$ $\left\{\vec{y} \in(Q(x, \delta))^{m}: \sum_{i=1}^{m}\left|x-y_{i}\right|^{2}>\delta^{2}\right\}$. It is clear that

$$
\begin{aligned}
\left|T_{\delta}(\vec{f})(x)\right| \leq & \left|\int_{U_{\delta}(x)} K(x, \vec{y}) \prod_{i=1}^{m} f_{i}\left(y_{i}\right) d y_{i}\right| \\
& +\left|\int_{\left((Q(x, \delta))^{m}\right)^{c}} K(x, \vec{y}) \prod_{i=1}^{m} f_{i}\left(y_{i}\right) d y_{i}\right| .
\end{aligned}
$$

By invoking the size condition (1.1), the first term on the right hand side of (2.2) can be estimated as follows:

$$
\begin{aligned}
\left|\int_{U_{\delta}(x)} K(x, \vec{y}) \prod_{i=1}^{m} f_{i}\left(y_{i}\right) d y_{i}\right| & \leq \int_{U_{\delta}(x)} \frac{A}{\left(\sum_{i=1}^{m}\left|y_{i}-x\right|\right)^{m n}} \prod_{i=1}^{m}\left|f_{i}\left(y_{i}\right)\right| d y_{i} \\
& \leq \int_{U_{\delta}(x)} \frac{C}{\delta^{m n}} \prod_{i=1}^{m}\left|f_{i}\left(y_{i}\right)\right| d y_{i} \\
& \leq \prod_{i=1}^{m} \frac{C}{(2 \delta)^{n}} \int_{Q(x, \delta)}\left|f_{i}\left(y_{i}\right)\right| d y_{i} \\
& \leq C \mathcal{M}(\vec{f})(x) .
\end{aligned}
$$

We now estimate the second term in 2.2. Pick $z \in Q(x, \delta / 2)$ and set $\overrightarrow{f_{0}}=\left(f_{1} \chi_{Q(x, \delta)}, \ldots, f_{m} \chi_{Q(x, \delta)}\right)$. Then

$$
\int_{\left((Q(x, \delta))^{m}\right)^{c}} K(z, \vec{y}) \prod_{i=1}^{m} f_{i}\left(y_{i}\right) d y_{i}=T(\vec{f})(z)-T\left(\vec{f}_{0}\right)(z),
$$

which means

$$
\begin{aligned}
& \left|\int_{\left((Q(x, \delta))^{m}\right)^{c}} K(x, \vec{y}) \prod_{i=1}^{m} f_{i}\left(y_{i}\right) d y_{i}\right| \\
& \leq\left|\int_{\left((Q(x, \delta))^{m}\right)^{c}} K(x, \vec{y}) \prod_{i=1}^{m} f_{i}\left(y_{i}\right) d y_{i}-\int_{\left((Q(x, \delta))^{m}\right)^{c}} K(z, \vec{y}) \prod_{i=1}^{m} f_{i}\left(y_{i}\right) d y_{i}\right| \\
& \quad+\left|T(\vec{f})(z)-T\left(\overrightarrow{f_{0}}\right)(z)\right| .
\end{aligned}
$$


In virtue of the smoothness condition 1.2 , we can deduce that

$$
\begin{aligned}
& \left|\int_{\left((Q(x, \delta))^{m}\right)^{c}} K(x, \vec{y}) \prod_{i=1}^{m} f_{i}\left(y_{i}\right) d y_{i}-\int_{\left((Q(x, \delta))^{m}\right)^{c}} K(z, \vec{y}) \prod_{i=1}^{m} f_{i}\left(y_{i}\right) d y_{i}\right| \\
& \leq \int_{\left((Q(x, \delta))^{m}\right)^{c}} \frac{C|x-z|^{\epsilon}}{\left(\sum_{i=1}^{m}\left|x-y_{i}\right|\right)^{m n+\epsilon}} \prod_{i=1}^{m}\left|f_{i}\left(y_{i}\right)\right| d y_{i} \\
& \leq \sum_{i_{1}, \ldots, i_{l}} \sum_{k=0}^{\infty} C \delta^{\epsilon} \prod_{i \in\left\{i_{1}, \ldots, i_{l}\right\}} \\
& \times \int_{Q(x, \delta)}\left|f_{i}\left(y_{i}\right)\right| d y_{i} \int_{\left(Q\left(x, 2^{k+1} \delta\right)\right)^{m-l} \backslash\left(Q\left(x, 2^{k} \delta\right)\right)^{m-l}} \frac{\prod_{i \notin\left\{i_{1}, \ldots, i_{l}\right\}}\left|f_{i}\left(y_{i}\right)\right| d y_{i}}{\left(\sum_{i=1}^{m}\left|x-y_{i}\right|\right)^{m n+\epsilon}} \\
& \leq \sum_{i_{1}, \ldots, i_{l}} \sum_{k=0}^{\infty} C \delta^{\epsilon} \prod_{i \in\left\{i_{1}, \ldots, i_{l}\right\}} \\
& \times \int_{Q(x, \delta)}\left|f_{i}\left(y_{i}\right)\right| d y_{i} \int_{\left(Q\left(x, 2^{k+1} \delta\right)\right)^{m-l}} \frac{\prod_{i \notin\left\{i_{1}, \ldots, i_{l}\right\}}\left|f_{i}\left(y_{i}\right)\right| d y_{i}}{\left(2^{k} \delta\right)^{m n+\epsilon}} \\
& \leq \sum_{k=0}^{\infty} \frac{C}{2^{k \epsilon}} \prod_{i=1}^{m} \frac{1}{\left(2^{k+2} \delta\right)^{n}} \int_{Q\left(x, 2^{k+1} \delta\right)}\left|f_{i}\left(y_{i}\right)\right| d y_{i} \\
& \leq C \mathcal{M} \vec{f}(x),
\end{aligned}
$$

where $\emptyset \neq\left\{i_{1}, \ldots, i_{l}\right\} \subsetneq\{1, \ldots, m\}$. This implies

$$
\begin{aligned}
&\left|\int_{\left((Q(x, \delta))^{m}\right)^{c}} K(x, \vec{y}) \prod_{i=1}^{m} f_{i}\left(y_{i}\right) d y_{i}\right| \\
& \leq C \mathcal{M} \vec{f}(x)+|T(\vec{f})(z)|+\left|T\left(\vec{f}_{0}\right)(z)\right| .
\end{aligned}
$$

Raising 2.3 to the power $\eta$, integrating over $z \in Q=Q(x, \delta / 2)$ and dividing by $|Q|$, we conclude that

$$
\begin{aligned}
\mid \int_{\left((Q(x, \delta))^{m}\right)^{c}} K(x, \vec{y}) & \left.\prod_{i=1}^{m} f_{i}\left(y_{i}\right) d y_{i}\right|^{\eta} \\
& \leq C(\mathcal{M} \vec{f}(x))^{\eta}+M\left(|T(\vec{f})|^{\eta}\right)(x)+\frac{1}{|Q|} \int_{Q}\left|T\left(\overrightarrow{f_{0}}\right)(z)\right|^{\eta} d z .
\end{aligned}
$$

Finally, the proof can be finished by using the arguments in [5] which proved $|Q|^{-1} \int_{Q}\left|T\left(\overrightarrow{f_{0}}\right)\right|^{\eta} \leq C\left(\prod_{i=1}^{m}|Q|^{-1} \int_{Q}\left|f_{i}\right|\right)^{\eta}$.

It is well known that $M$ is bounded from $L^{p}(\omega)$ to $L^{p, \infty}(\omega)$ when $\omega \in A_{p}$ and $p \geq 1$. Similar to the proof in [2, p. 135], we get the following lemma to show the weak estimates in the main theorem. 
Lemma 2.2. If $\omega \in A_{p}$ and $p \geq 1$, then $M$ maps $L^{p, \infty}(\omega)$ to $L^{p, \infty}(\omega)$.

Proof. For any cube $Q$ and $\omega \in A_{p}$,

$$
\begin{aligned}
&\left(\frac{1}{|Q|} \int_{Q} \omega\right)\left(\frac{\lambda}{|Q|} \int_{Q \cap\{|f|>\lambda\}}\right)^{p} \\
& \leq\left(\frac{1}{|Q|} \int_{Q} \omega\right)\left(\frac{\lambda^{p}}{|Q|} \int_{Q \cap\{|f|>\lambda\}} \omega\right)\left(\frac{1}{|Q|} \int_{Q} \omega^{1-p^{\prime}}\right)^{p-1} \\
& \leq C \frac{\lambda^{p}}{|Q|} \int_{Q \cap\{|f|>\lambda\}} \omega .
\end{aligned}
$$

When $p=1,\left(|Q|^{-1} \int_{Q} \omega^{1-p^{\prime}}\right)^{p-1}$ is understood as $\left(\inf _{Q} \omega\right)^{-1}$. Then we obtain

$$
\begin{aligned}
\int_{Q} \omega & \leq C\left(\frac{\lambda}{|Q|} \int_{Q \cap\{|f|>\lambda\}}\right)^{-p}\left(\lambda^{p} \int_{Q \cap\{|f|>\lambda\}} \omega\right) \\
& \leq C\left(\frac{1}{|Q|} \int_{Q}|f|\right)^{-p}\left(\lambda^{p} \int_{Q \cap\{|f|>\lambda\}} \omega\right) .
\end{aligned}
$$

A Calderón-Zygmund decomposition for $f$ at height $4^{-n} \lambda$ yields a sequence of cubes $\left\{Q_{k}\right\}$ such that $4^{-n} \lambda<\left|Q_{k}\right|^{-1} \int_{Q_{k}} f$. Additionally, we have $\{M f>\lambda\} \subset \bigcup_{k} 3 Q_{k}$ as in [2]. Since the function $\omega \in A_{p}$ is doubling, it is immediate that

$$
\begin{aligned}
\int_{\{M f>\lambda\}} \omega & \leq \sum_{k} \int_{3 Q_{k}} \omega \leq C 3^{n p} \sum_{k} \int_{Q_{k}} \omega \\
& \leq C 3^{n p} \sum_{k}\left(\frac{1}{\left|Q_{k}\right|} \int_{Q_{k}}|f|\right)^{-p}\left(\lambda^{p} \int_{Q_{k} \cap\{|f|>\lambda\}} \omega\right) \leq C 12^{n p} \int_{\{|f|>\lambda\}} \omega,
\end{aligned}
$$

which means $\|M f\|_{L^{p, \infty}(\omega)} \leq C\|f\|_{L^{p, \infty}(\omega)}$.

Proof of Theorem 1.3. Before the final proof, we should recall another fact from [6]: if $\vec{\omega} \in A_{\vec{p}}$, then $\nu_{\vec{\omega}} \in A_{m p}$.

When $1 \leq p_{1}, \ldots, p_{m}<\infty$, we have a weak type result. Let $\eta \leq 1 / m$. The improved Cotlar inequality (2.1), Lemma 2.2 and the weighted estimates (1.6) for $\mathcal{M}$ and $T$ in [6] imply

$$
\begin{aligned}
& \left\|T^{*}(\vec{f})\right\|_{L^{p, \infty}\left(\nu_{\vec{\omega}}\right)} \leq C\left(\left\|M_{\eta}(T(\vec{f}))\right\|_{L^{p, \infty}\left(\nu_{\vec{\omega}}\right)}+\|\mathcal{M}(\vec{f})\|_{L^{p, \infty}\left(\nu_{\vec{\omega}}\right)}\right) \\
& =C\left(\left\|M\left(|T(\vec{f})|^{\eta}\right)\right\|_{L^{p / \eta, \infty\left(\nu_{\vec{\omega}}\right)}}^{1 / \eta}+\|\mathcal{M}(\vec{f})\|_{L^{p, \infty}\left(\nu_{\vec{\omega}}\right)}\right) \\
& \leq C\left(\left\||T(\vec{f})|^{\eta}\right\|_{L^{p / \eta, \infty}\left(\nu_{\vec{\omega}}\right)}^{1 / \eta}+\|\mathcal{M}(\vec{f})\|_{L^{p, \infty}\left(\nu_{\vec{\omega}}\right)}\right) \\
& =C\left(\|T(\vec{f})\|_{L^{p, \infty}\left(\nu_{\vec{\omega}}\right)}+\|\mathcal{M}(\vec{f})\|_{L^{p, \infty}\left(\nu_{\vec{\omega}}\right)}\right) \leq C \prod_{i=1}^{m}\left\|f_{i}\right\|_{L^{p_{i}\left(\omega_{i}\right)}} .
\end{aligned}
$$

The proof of the strong case is similar. 
3. Further results. X. T. Duong, R. Gong, L. Grafakos, J. Li and L. Yan [3] studied the maximal operator of a multilinear singular integral with non-smooth kernel. Together with the corresponding Cotlar inequalities with $\prod_{i=1}^{m} M f_{i}$ and unweighted bounds, they obtained the counterpart of Theorem 1.2 in that case. However, weighted norm inequalities with new multiple weights for non-smooth operators as we did for Calderón-Zygmund operators have not been proved yet.

Acknowledgements. The author would like to thank the referee for his or her helpful and kind comments.

\section{References}

[1] R. R. Coifman and C. Fefferman, Weighted norm inequalities for maximal functions and singular integrals, Studia Math. 51 (1974), 241-250.

[2] J. Duoandikoetxea, Fourier Analysis, Grad. Stud. Math. 29, Amer. Math. Soc., Providence, RI, 2001.

[3] X. T. Duong, R. Gong, L. Grafakos, J. Li, and L. Yan, Maximal operator for multilinear singular integrals with non-smooth kernels, Indiana Univ. Math. J. 58 (2009), 2517-2542.

[4] L. Grafakos and R. H. Torres, Multilinear Calderón-Zygmund theory, Adv. Math. 165 (2002), 124-164.

[5] - - - Maximal operator and weighted norm inequalities for multilinear singular integrals, Indiana Univ. Math. J. 51 (2002), 1261-1276.

[6] A. K. Lerner, S. Ombrosi, C. Pérez, R. H. Torres and R. Trujillo-González, New maximal functions and multiple weights for the multilinear Calderón-Zygmund theory, Adv. Math. 220 (2009), 1222-1264.

\section{Xi Chen}

School of Mathematical Sciences

Beijing Normal University

Laboratory of Mathematics and Complex Systems

Ministry of Education

Beijing 100875

People's Republic of China

E-mail: x.chen@mail.bnu.edu.cn

Received February 13, 2009;

received in final form July 23, 2010 